\title{
Avaliação ultra-sonográfica do segmento posterior de olhos de cães diabéticos e não diabéticos portadores de catarata ${ }^{1}$
}

\author{
Renata Squarzoni ${ }^{2 *}$, Maira S.A. Morales ${ }^{3}$, Angélica M.V. Safatle ${ }^{4}$ e \\ Paulo Sérgio M. Barros ${ }^{5}$
}

\begin{abstract}
Squarzoni R., Morales M., Safatle A.M.V. \& Barros P.S.M. 2007. [Ultrasonographic evaluation of the eyes' posterior segment of diabetic and non-diabetic dogs with cataracts.] Avaliação ultra-sonográfica do segmento posterior de olhos de cães diabéticos e não diabéticos portadores de catarata. Pesquisa Veterinária Brasileira 27(11):455-461. Departamento de Cirurgia, Faculdade de Medicina Veterinária e Zootecnia (FMVZ), Universidade de São Paulo (USP), Av. Orlando Marques de Paiva 87, São Paulo, SP 05508-900, Brazil. E-mail: renatasq@usp.br

Cataracts jeopardize the light penetration into the eye and may restrain direct exam of eye structures. It is necessary to identify very early any eye disease that could cause the failure of cataract surgery. The ocular ultrasonography or echography is a safe, non invasive, inexpensive and easy procedure that complements ocular examination. Two hundred and twenty five eyes of 123 dogs were evaluated using a $10 \mathrm{Mhz}$ linear transducer ultrasound. The dogs were divided into three groups, as there is Group 1: 36 healthy control dogs; Group 2: 52 non diabetic dogs with cataracts; and Group 3: 35 diabetic dogs with cataracts; all of them were patients of the Veterinary Teaching Hospital, University of Sao Paulo. The ultrasonographic findings were in Group 1: vitreous degeneration in $43 \%$, posterior vitreous detachment in $7.7 \%$, asteroid hyalosis in $4.6 \%$ of the eyes; in Group 2: vitreous degeneration in $58.9 \%$, posterior vitreous detachment in $8.4 \%$, asteroid hyalosis in $2.1 \%$, inflammatory or haemorrhagic process in $2.1 \%$, retinal detachment in $4.3 \%$ of the eyes; and in Group 3: vitreous degeneration in $50.7 \%$, posterior vitreous detachment in $13.8 \%$, asteroid hyalosis in $12.3 \%$, inflammatory or haemorrhagic process in $3.1 \%$, retinal detachment in $3.1 \%$ of the eyes. It could be concluded that (1) diabetic dogs, mainly the ones with diabetes for more than 2 years, have greater chances to develop asteroid hyalosis; (2) there were no significant differences in retinal detachment frequencies for the diabetic and non diabetic dogs; and (3) the ocular ultrasonography is a safe, effective and important exam for the pre-operatory evaluation of dogs with cataracts.
\end{abstract}

INDEX TERMS: Ocular ultrasound, ocular echography, Diabetes mellitus, cataracts, retinal detachment.

RESUMO.- A catarata impossibilita a passagem de luz dentro do olho, dificultando o exame físico direto das estruturas oculares. É necessária a identificação prévia de quaisquer al-

\footnotetext{
${ }^{1}$ Recebido em 14 de maio de 2007.

Aceito para publicação em 20 de junho de 2007.

2 Pós-Graduanda, Departamento de Cirurgia da Faculdade de Medicina Veterinária e Zootecnia (FMVZ), Universidade de São Paulo (USP), Av. Orlando Marques de Paiva 87, São Paulo, SP 05508-900, Brasil. "Autor para correspondência: renatasq@usp.br

${ }^{3}$ Setor de Ultra-Som Ocular do Departamento de Oftalmologia, Universidade Federal de São Paulo (UNIFESP), Escola Paulista de Medicina (EPM), Rua Botucatu 822, São Paulo, SP 04023-062, Brasil.

${ }^{4}$ Serviço de Oftalmologia, Hospital Veterinário, USP, Av. Orlando Marques de Paiva 87, São Paulo, SP

${ }^{5}$ Departamento de Cirurgia, FMVZ, USP, São Paulo, SP.
}

terações oculares que contra-indiquem a cirurgia de catarata. A ultra-sonografia, ou ecografia ocular, é um exame seguro, de baixo custo, não invasivo, de fácil realização, que completa o exame oftálmico. Foi realizada avaliação ultra-sonográfica, com transdutor linear, frequiência de $10 \mathrm{MHz}$, de 225 olhos de 123 cães, sendo cães controle (Grupo 1), 52 cães não diabéticos portadores de catarata (Grupo 2) e 35 cães diabéticos portadores de catarata (Grupo 3), todos eles atendidos no Hospital Veterinário da Universidade de São Paulo. Foram encontradas as seguintes alterações ultra-sonográficas no Grupo 1: degeneração vítrea em $43 \%$, descolamento de vítreo posterior em $7,7 \%$, hialose asteróide em 4,6\% dos olhos; no Grupo 2: degeneração vítrea em $58,9 \%$, descolamento de vítreo posterior em $8,4 \%$, hialose asteróide em $2,1 \%$, processo inflamatório e/ou hemorrágico em $2,1 \%$ e descolamento de 
retina em 4,3\% dos olhos; e no Grupo 3: degeneração vítrea em $50,7 \%$, descolamento de vítreo posterior em $13,8 \%$, hialose asteróide em 12,3\%, processo inflamatório e/ou hemorrágico em $3,1 \%$ e descolamento de retina em $3,1 \%$ dos olhos. Concluiu-se, que (1) cães diabéticos, principalmente os que apresentam a doença há mais de 2 anos, tem maior predisposição para o desenvolvimento de hialose asteróide; (2) não houve diferença entre a freqüencia de descolamento de retina encontrada em cães diabéticos e não diabéticos; e (3) a ultrasonografia ocular é em exame seguro, eficaz e importante na avaliação pré-operatória de cães portadores de catarata.

TERMOS DE INDEXAÇÃO: Ultra-som ocular, ecografia ocular, diabetes melito, catarata, descolamento de retina.

\section{INTRODUÇÃO}

A cirurgia de catarata em cães está cada vez mais acessível em nosso meio. Porém, como a opacificação do cristalino muitas vezes impede a avaliação pré-cirúrgica do segmento posterior do olho, torna-se necessário o uso de exames complementares que possibilitem essa avaliação. Qualquer tipo de alteração em segmento posterior de olhos de cães portadores de catarata deve ser identificado antes da cirurgia. Descolamentos de retina, hemorragia ou processo inflamatório vítreo, por exemplo, contra-indicam a facectomia. A ultrasonografia ocular é um exame prático, barato, fácil de ser realizado, não invasivo, que permite estudo anatômico confiável do segmento posterior, sempre que houver qualquer opacidade de meios que impossibilite o exame oftalmoscópico.

Embora muitas tenham sido as publicações sobre a ultrasonografia ocular e grande tenha sido o seu desenvolvimento antes de Ossoinig (1979), foi com este pesquisador que o exame ecográfico consolidou-se como um método não invasivo, inócuo e, hoje, indispensável como parte da propedêutica armada em oftalmologia.

Em 1997, foi realizado por Van der Woerdt estudo retrospectivo sobre anormalidades ultra-sonográficas nos olhos de 147 cães com catarata, no qual 23\% dos olhos apresentaram degeneração vítrea e $11 \%$ apresentaram descolamento de retina. Outro estudo semelhante foi realizado por Anteby, em 1998, em 509 pacientes humanos que apresentavam catarata total. Neste estudo, 19,6\% dos pacientes submetidos ao exame ultra-sonográfico antes da cirurgia de facectomia apresentaram alterações do segmento posterior, entre elas, estafiloma posterior $(7,2 \%)$, descolamento de retina $(4,5 \%)$ e hemorragia vítrea $(2,5 \%)$. Entende-se, portanto, que o exame ecográfico é de suma importância no diagnóstico de doenças do segmento posterior, uma vez que existam opacidades de córnea, câmara anterior ou cristalino que impeçam o exame direto das estruturas oculares.

Em cães diabéticos, a catarata é a manifestação ocular mais freqüente e sua incidência é muito alta, chegando a 68\% (38 de 56 cães diabéticos), conforme estudo realizado em 1960, por Wilkinson. As alterações vítreas no paciente diabético estão intimamente relacionadas à doença retiniana em humanos. Em cães, estudos sobre a existência de vitreopatia diabética ainda não foram realizados. Além da vitreopatia diabética, alguns estudos provaram associação de hialose asteróide com diabetes melito. Bergren (1991) identificou a presença de diabetes melito em 29 de 101 pacientes com hialose asteróide examinados, em comparação a 10 de 101 controles. A retinopatia diabética é, sem dúvida, a alteração ocular mais importante no diabetes melito em seres humanos, e está associada com alterações em vasos sanguíneos pequenos em todo o organismo, inclusive na retina. $O$ primeiro relato da doença em um cão foi documentado por Patz \& Maumenee (1962). Porém, a retinopatia diabética não é comum em cães. A principal explicação para este fato é o tempo que a doença leva para manifestar-se após o diagnóstico do diabetes melito, geralmente anos. Os cães, normalmente, apresentam curta expectativa de vida (2-5 anos) após o diagnóstico do diabetes (Feldman 1987). Com o avanço da medicina veterinária, os animais diabéticos estão vivendo mais e é provável que essas complicações tardias irão apresentar-se com maior frequiência. A extensão e a severidade da retinopatia diabética em cães são extremamente leves se comparadas à observada em seres humanos. A maioria dos casos é subclínica e não foi reportado nenhum caso de perda de visão em cães, atribuída aos efeitos dessa complicação. Sibay (1967) sugeriu que a diabetes precisa estar presente por pelo menos cinco anos no cão, antes da manifestação da retinopatia. Existem vários casos reportados de retinopatia em cães com diabetes melito espontânea (Patz 1962,1965, Sibay 1967, Monti 1976, Barnett 1981, Ono 1986). Recentemente, em 2003, Landrey realizou estudo retrospectivo, em que avaliou 52 cães diabéticos e 179 cães não diabéticos submetidos à cirurgia de catarata. Dos 52 cães diabéticos, $11(21,1 \%)$ desenvolveram sinais oftalmoscópicos de retinopatia diabética após cirurgia de catarata. $O$ período médio entre o diagnóstico de diabetes e o aparecimento da retinopatia foi de 1,4 anos $(0,5-$ 3,2 anos). Alterações retinianas foram encontradas em apenas $0,6 \%$ dos animais não diabéticos. Como a prevalência de catarata em cães diabéticos é muito alta, sendo a avaliação do segmento ocular posterior inadequada, há dificuldade de identificação de qualquer doença vítreo-retiniana nesses animais.

Esse trabalho tem por objetivo a avaliação ultra-sonográfica das estruturas intra-oculares de cães diabéticos e não diabéticos, portadores de catarata, para a detecção de alterações de segmento posterior que possam contra-indicar o procedimento cirúrgico desses olhos.

\section{MATERIAL E MÉTODOS}

O protocolo de estudo foi aprovado pela Comissão de Bioética da Faculdade de Medicina Veterinária e Zootecnia da Universidade de São Paulo.

Foram avaliados 123 cães (225 olhos) atendidos no Hospital Veterinário da Faculdade de Medicina Veterinária (HOVET), Universidade de São Paulo, e subdivididos em três grupos: 36 cães, machos ou fêmeas, portadores de enfermidades não oculares, usados como grupo controle (Grupo 1); 52 cães não diabéticos, machos ou fêmeas, portadores de catarata juvenil ou senil, uni ou bilateral, candidatos à cirurgia de catarata (Grupo 2); e 35 cães diabéticos, 
machos ou fêmeas, portadores de catarata uni ou bilateral (Grupo 3). Os animais foram submetidos a exame oftalmológico, incluindo biomicroscopia com lâmpada de fenda (Kowa, md SL-14), tonometria de aplanação (Tonopen, Mentor, mod. XL), oftalmoscopia direta (Welsh Allyn) e indireta (Neitz, mod. IO-H), quando possível, por causa da catarata. Entre os dados coletados e anotados em fichas individuais, estavam raça, idade, sexo, classificação da catarata (imatura, matura e hipermatura), tempo de evolução da catarata (dividido em menos de 6 meses, de 6-24 meses e acima de 24 meses de evolução), tempo de evolução do diabetes (dividido em até 6 meses, de 6-24 meses e acima de 24 meses), olho examinado e classificação das alterações ultra-sonográficas encontradas em segmento posterior.

Foram utilizados, como critérios de inclusão para o Grupo 1: animais sem alterações oculares; Grupo 2: cães não diabéticos portadores de catarata imatura, matura ou hipermatura; e para o Grupo 3: diabetes melito diagnosticada por valores glicêmicos acima do limite normal superior para cães, em jejum; catarata imatura, matura ou hipermatura, uni ou bilateral.

Foram definidos, como critérios de exclusão para o Grupo 1: idade inferior a um ano; hipertensão arterial sistêmica ou qualquer outra doença sistêmicica que possa ser relacionada com alterações oculares; história de trauma ou cirurgia ocular prévia e qualquer doença ocular aparente ou previamente conhecida; Grupo 2: idade inferior a um ano; hipertensão arterial sistêmica ou qualquer outra doença sistêmica que possa ser relacionada com alterações oculares; história de trauma ou cirurgia ocular prévia e qualquer doença ocular, com exceção da catarata, aparente ou previamente conhecida; e para o Grupo 3: idade inferior a um ano; hipertensão arterial sistêmica; hiperadrenocorticismo; glaucoma ou atrofia bulbar; história de trauma ou cirurgia ocular prévia e doenças oculares previamente conhecidas do segmento posterior.

A avaliação do segmento posterior do olho, utilizando-se aparelho de ultra-som (Zub - 405 Plus, Hitachi) ${ }^{5}$ foi realizada com os animais alertas, apenas com anestesia tópica oftálmica à base de proxi-metacaína a $0,5 \%$. Não foi necessária tranqüilização em nenhum exame. Os animais foram contidos manualmente e posicionados sentados ou em decúbito esternal. Foi utilizada a técnica de contato corneal, com o uso de gel hidrofilico próprio para ultra-som (Carbogel - ULT, Carbogel Ind. e Com. Ltda) entre a córnea e a sonda. Foram realizados exames ultra-sonográficos, no modo $\mathrm{B}$, com transdutor de estado sólido de $10 \mathrm{MHz}$. Após o exame, os olhos foram cuidadosamente enxaguados com solução fisiológica para a remoção do gel. Os exames foram documentados por meio de impressora a laser (Vídeo Graphic Printer UP-895MD, Sony) e as imagens, revisadas para estudo.

Para as variáveis qualitativas, foram utilizadas as frequiências relativas (percentuais) e a frequiência absoluta $(\mathrm{N})$ das classes de cada variável qualitativa. Para as variáveis quantitativas foram utilizadas médias e medianas, para resumir as informações, e desvios padrão, mínimo e máximo para indicar a variabilidade dos dados. Na análise estatística, para as variáveis qualitativas, foi utilizado o teste do Quiquadrado, para verificar possíveis associações entre as classes das características dos animais. Foi considerado haver associações entre as classes das variáveis quando o resultado do teste apresentou nível descritivo abaixo de 0,05 (valor de $p<0,05$ ).

\section{RESULTADOS}

\section{Cães controle (Grupo 1)}

Foram examinados 65 olhos, sendo 33 olhos esquerdos e 32 olhos direitos de um total de 36 cães, 13 machos e 23

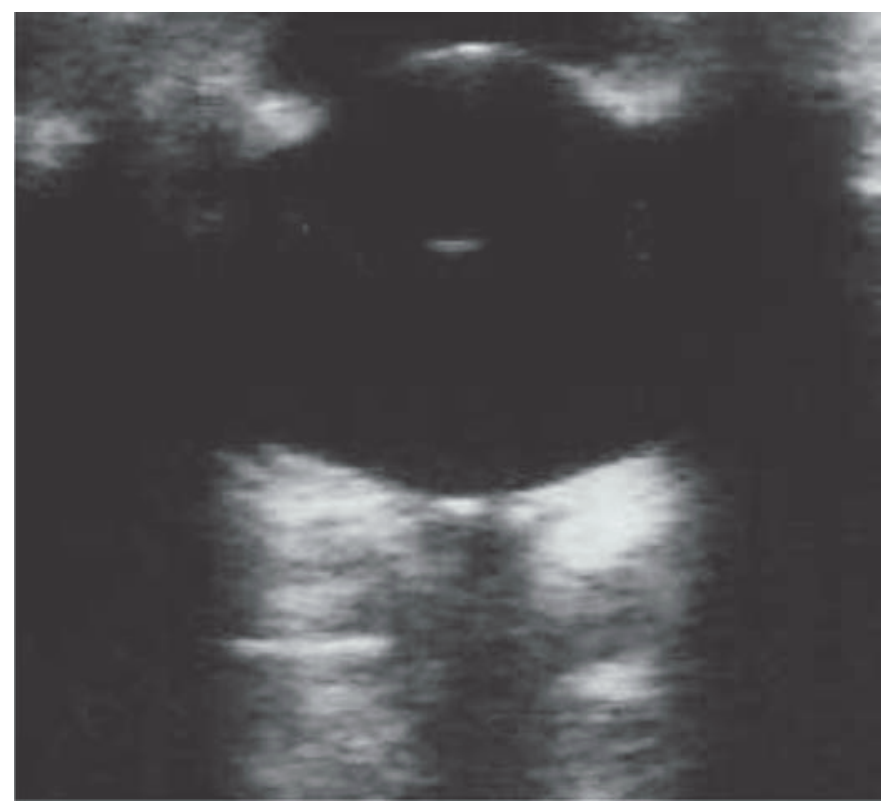

Fig.1. Exame ultra-sonográfico normal, corte axial horizontal, do olho esquerdo do cão da raça Terrier Brasileiro, fêmea, 12 anos, do Grupo 1.

fêmeas. Vinte e nove olhos apresentaram exame ultrasonográfico normal (Fig.1). Foram observadas membranas vítreas sugestivas de degeneração vítrea em 28 olhos (43\%), sendo $27(41,5 \%)$ em pequena quantidade e um $(1,5 \%)$ em quantidade moderada. Descolamento de vítreo posterior foi observado em cinco olhos $(7,7 \%)$. Hialose asteróide foi observada em três olhos $(4,6 \%)$.

No cruzamento entre as faixas etárias e as alterações ultrasonográficas encontradas neste grupo, foi observado um maior percentual de descolamento de vítreo posterior entre os animais com idade superior a 11 anos.

\section{Cães não diabéticos com catarata (Grupo 2)}

Noventa e cinco olhos foram examinados, sendo 51 olhos esquerdos e 44 olhos direitos, de 52 animais portadores de catarata uni ou bilateral, 26 machos e 26 fêmeas. Todos esses olhos apresentavam catarata, classificada em imatura (20\%), matura $(25,3 \%)$, hipermatura $(54,7 \%)$. 0 tempo de evolução da catarata variou entre 2 e 72 meses, com média de 16,5 meses de evolução. De acordo com a classificação adotada: $32,6 \%$ dos olhos apresentavam catarata há menos de 6 meses, 53,7\% entre 6 e 24 meses e 13,7\% há mais de 24 meses. Trinta olhos apresentaram exame ultra-sonográfico normal, com exceção da catarata. Degeneração vítrea foi encontrada em 56 olhos $(58,9 \%)$, sendo 39 olhos $(41,1 \%)$ em grau leve, $15(15,8 \%)$ em grau moderado e dois $(2,1 \%)$ em grau severo. Descolamento de vítreo posterior foi encontrado em oito olhos $(8,4 \%)$. Hialose asteróide foi observada em dois olhos $(2,1 \%)$. Foi notada presença de ecos puntiformes em grande quantidade, sugerindo processo inflamatório e/ou hemorrágico, em dois olhos de um mesmo animal, cujo hemograma revelou trombocitopenia $\left(70.000 .000\right.$ plaquetas $\left./ \mathrm{mm}^{3}\right)$. Descolamento de retina foi encontrado em quatro olhos $(4,3 \%)$. 


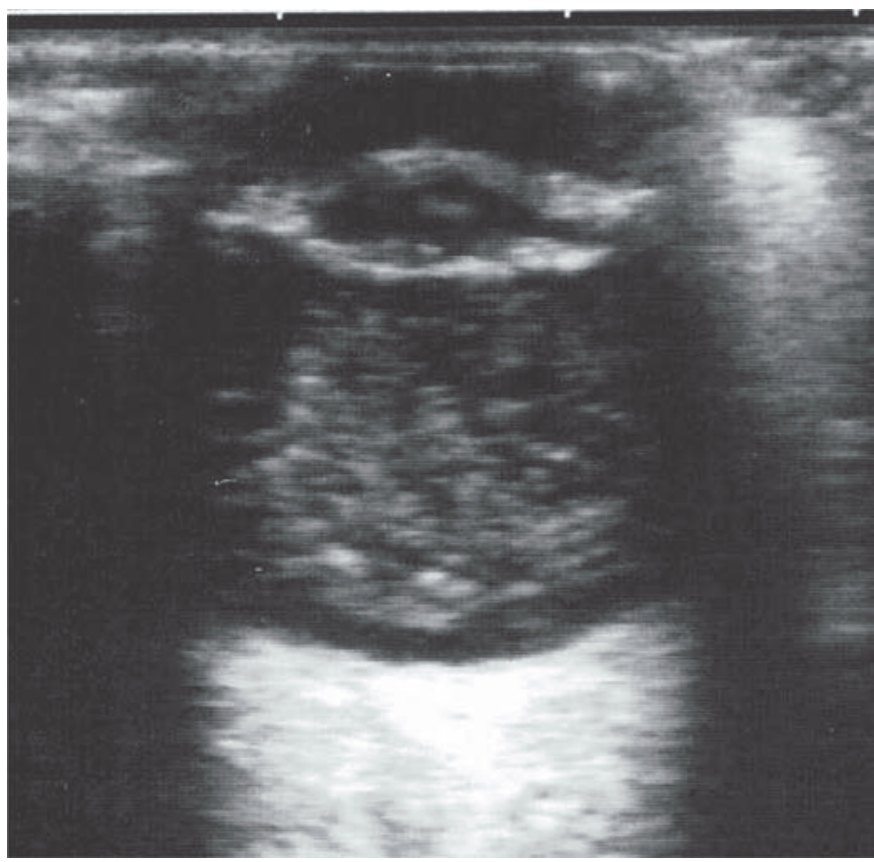

Fig.2. Exame ultra-sonográfico, corte axial horizontal, mostrando hiperecogenicidade do cristalino, hialose asteróide no olho direito do cão da raça Dachshund, fêmea, 14 anos, do Grupo 3.

A comparação entre as alterações ultra-sonográficas e o grau ou tempo de evolução da catarata não mostrou nenhuma relação significante.

\section{Cães diabéticos com catarata (Grupo 3)}

Foram examinados 65 olhos, sendo 33 olhos esquerdos e 32 olhos direitos de 35 animais portadores de catarata uni ou bilateral, quatro machos e 31 fêmeas. Todos esses olhos apresentavam catarata, classificada em imatura $(3,1 \%)$, matura $(9,2 \%)$, hipermatura $(87,3 \%)$. O tempo de evolução da catarata variou entre 1 e 60 meses, com média de 17,6 meses de evolução. Dos olhos examinados, 33,3\% apresentava catarata há menos de 6 meses, $41,3 \%$ entre 6 e 24 meses e 25,4\% há mais de 24 meses de evolução. Dos animais avaliados, 30,8\% apresentava diabetes há menos de 6 meses, 41,5\% entre 6 e 24 meses e $27,7 \%$ há mais de 24 meses.

Vinte e dois olhos apresentaram exame ultra-sonográfico normal, com exceção da catarata. Degeneração vítrea foi encontrada em 33 olhos $(50,7 \%)$, sendo 26 olhos $(40,0 \%$ ) em grau leve, seis $(9,2 \%)$ em grau moderado e um $(1,5 \%)$ em grau severo. Descolamento de vítreo posterior foi encontrado em nove olhos $(13,8 \%)$. Hialose asteróide (Fig.2) foi observada em oito olhos $(12,3 \%)$. Foi observada presença de ecos puntiformes, sugerindo processo inflamatório e/ou hemorrágico em dois olhos $(3,1 \%)$. Descolamento de retina (Fig.3) foi encontrado em dois olhos $(3,1 \%)$.

No cruzamento entre as faixas etárias e as alterações ultrasonográficas encontradas neste grupo, observou-se um maior percentual de portadores de hialose asteróide entre os animais com idade inferior a 4 anos; foi notado um maior percentual de cães com descolamento de vítreo posterior entre os animais com idade inferior a 4 anos.

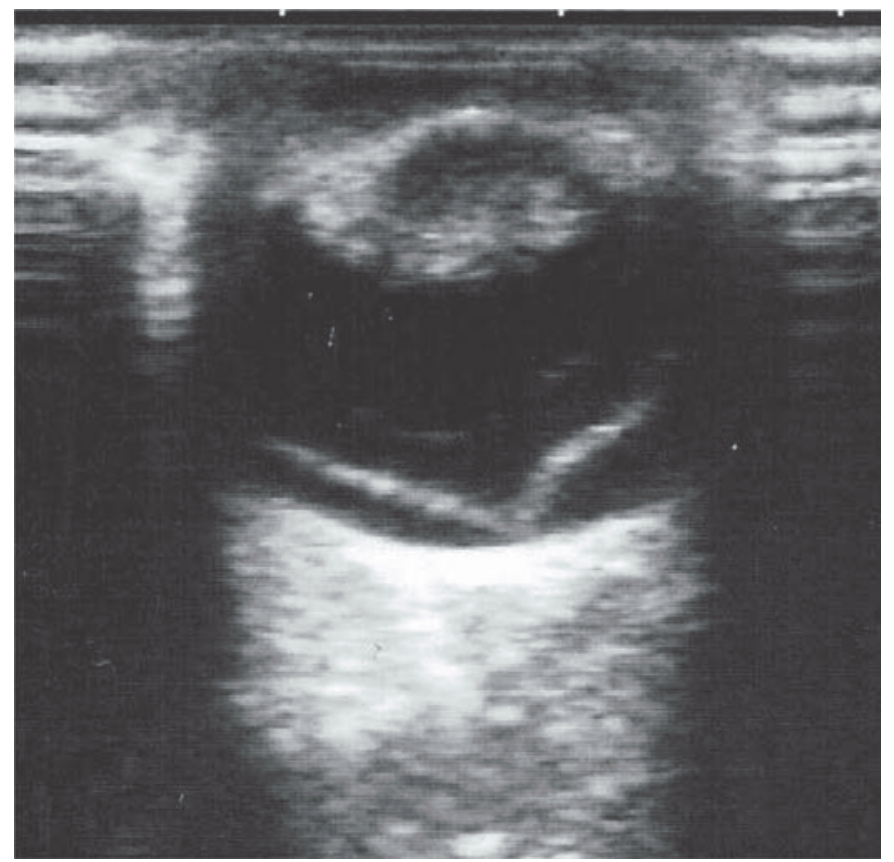

Fig.3. Exame ultra-sonográfico, corte transversal às 6 horas, mostrando hiperecogenicidade do cristalino e descolamento de retina total no olho direito do cão da raça Poodle, fêmea, 5 anos, do Grupo 2.

A relação entre o tempo de evolução da catarata e os resultados dos exames ultra-sonográficos obtidos mostrou um maior percentual de animais com hialose asteróide entre aqueles com tempo de evolução da catarata acima de 2 anos. Notou-se, também, um maior percentual de animais com ausência de DVP entre os cães com até seis meses de evolução da catarata e um maior percentual de DVP total entre aqueles com tempo de evolução da catarata acima de 2 anos.

O tempo de evolução do diabetes foi relacionado com os achados ultra-sonográficos e observou-se maior percentual de animais com hialose asteróide entre aqueles com tempo de evolução do diabetes acima de 2 anos. Houve, também, maior percentual de animais com ausência de DVP entre os cães com até 6 meses de evolução do diabetes e um maior percentual de DVP total entre aqueles com tempo de evolução do diabetes acima de 2 anos.

No cruzamento entre o grau de catarata e os achados ultrasonográficos, encontrou-se maior percentual de degeneração vítrea moderada ou severa entre os animais que apresentaram catarata matura.

Encontrou-se associação estatisticamente significante entre os tempos de evolução do diabetes e da catarata. De acordo com a análise realizada, observou-se que quanto maior o tempo de evolução do diabetes, maior o tempo de evolução da catarata.

\section{Comparação entre os grupos}

Pelos resultados do teste do Qui-quadrado, observou-se associação estatisticamente significante entre as distribuições da variável sexo com os grupos $(p>0,05)$. Houve um maior percentual de animais do sexo feminino no grupo com catarata e diabetes. 
Quadro 1. Freqüencia e porcentagem dos olhos, direito e esquerdo, e alterações ultrasonográficas em segmento posterior dos cães dos três grupos (1, 2 e 3) e seus respectivos níveis descritivos

\begin{tabular}{|c|c|c|c|c|c|c|c|c|}
\hline & & \multicolumn{6}{|c|}{ Grupo $^{\mathrm{a}}$} & \multirow{3}{*}{$\begin{array}{l}\text { Nível des- } \\
\text { critivo (va- } \\
\text { lor de } p \text { ) }\end{array}$} \\
\hline & & \multicolumn{2}{|c|}{ 1) Controle } & \multicolumn{2}{|c|}{ 2) Com catarata } & \multicolumn{2}{|c|}{$\begin{array}{l}\text { 3) Com catara- } \\
\text { ta e diabetes }\end{array}$} & \\
\hline & & $\mathrm{N}$ & $\%$ & $\mathrm{~N}$ & $\%$ & $\mathrm{~N}$ & $\%$ & \\
\hline \multirow[t]{2}{*}{ Olho } & Esquerdo & 33 & $50,8 \%$ & 51 & $53,7 \%$ & 33 & $50,8 \%$ & 0,911 \\
\hline & Direito & 32 & $49,2 \%$ & 44 & $46,3 \%$ & 32 & $49,2 \%$ & \\
\hline \multirow[t]{2}{*}{ Asteróides hialóides } & Ausente & 62 & $95,4 \%$ & 93 & $97,9 \%(1)$ & 57 & $87,7 \%$ & 0,022 \\
\hline & Presente & 3 & $4,6 \%$ & 2 & $2,1 \%$ & 8 & $12,3 \%(2)$ & \\
\hline DVP - descolamento & Presente & 5 & $7,7 \%$ & 8 & $8,4 \%$ & 9 & $13,8 \%$ & 0,419 \\
\hline de vítreo posterior & Ausente & 60 & $92,3 \%$ & 87 & $91,6 \%$ & 56 & $86,2 \%$ & \\
\hline \multirow[t]{3}{*}{ Descolamento de retina } & Ausente & 65 & $100,0 \%$ & 91 & $95,8 \%$ & 63 & $96,9 \%$ & 0,260 \\
\hline & Presente & & & 4 & $4,3 \%$ & 2 & $3,1 \%$ & \\
\hline & Leve & 27 & $41,5 \%$ & 39 & $41,1 \%$ & 26 & $40,0 \%$ & \\
\hline \multirow[t]{3}{*}{ Degeneração vítrea } & Moderada & 1 & $1,5 \%$ & 15 & $15,8 \%$ & 6 & $9,2 \%$ & 0,076 \\
\hline & Severa & & & 2 & $2,1 \%$ & 1 & $1,5 \%$ & \\
\hline & Ausente & 37 & $56,9 \%$ & 39 & $41,1 \%$ & 32 & $49,2 \%$ & \\
\hline
\end{tabular}

a Valores de Z-res (Resíduo): 1) 2,0; 2) 2,7.

A análise estatística utilizada para comparação da idade entre os grupos mostrou que houve diferença significante entre eles, sendo o grupo de animais diabéticos (média de 10,3 anos de idade) mais velho do que os outros dois grupos (média de 8,2 anos de idade).

Comparando-se as alterações ultra-sonográficas entre os três grupos, observou-se maior percentual hialose asteróide no grupo com catarata e diabetes (Quadro 1).

Obteve-se, na comparação entre os grupos com catarata e com catarata e diabetes, um maior percentual de animais com catarata imatura ou matura no grupo com catarata, e um maior percentual de animais com catarata hipermatura no grupo com diabetes.

\section{DISCUSSÃO}

Com o avanço da cirurgia de catarata em cães e o interesse do médico veterinário por uma maior previsibilidade dos resultados visuais, é de suma importância a detecção de outras alterações oculares nos pacientes portadores de catarata. Nestes casos, a ultra-sonografia ocular é o exame mais utilizado para o diagnóstico de eventuais alterações anatômicas do segmento posterior que possam comprometer o resultado visual pós-operatório.

Até o fim da década de 60, era esperado que o corpo vítreo do olho saudável fosse ultra-sonograficamente homogêneo. No início da década de 70, vários estudos ultrasonográficos em seres humanos demonstraram que o vítreo sofre evidente degeneração de acordo com o aumento da idade (Rossi \& Gallenga 1971, Oksala 1975, 1978). No estudo atual, não se observou diferença estatisticamente significante entre idade e degeneração vítrea em nenhum dos grupos, porém é evidente a menor porcentagem desta alteração em animais com menos de 4 anos de idade. Em nenhum caso, a degeneração vítrea encontrada constituiu fator de contra-indicação cirúrgica. Nossos achados em pacientes caninos são compatíveis com os dados encontrados em pacientes humanos. Acreditamos que, assim como acontece em humanos, a degeneração vítrea encontrada em cães idosos pode ser considerada uma alteração senil fisiológica.

A membrana hialóde posterior, não impregnada por sangue ou células inflamatórias, mostra-se como eco com alta mobilidade e baixa reflexibilidade; se aderida, tem sua mobilidade diminuída. No grupo controle, os animais acima de 11 anos de idade foram os que apresentaram DVP com maior frequiência. No grupo com catarata, não foi encontrado DVP em olhos com catarata imatura, apenas nos olhos com catarata matura ou hipermatura. Porém, essa diferença não foi significante a ponto de se estabelecer uma relação entre DVP e grau da catarata. Apesar de os animais diabéticos apresentarem uma maior porcentagem de DVP, esta diferença não foi estatisticamente significante. Os animais com tempo de evolução do diabetes maior que 2 anos foram os que apresentaram maior porcentagem de DVP total, todos eles com catarata hipermatura. Em estudo postmortem realizado em pacientes humanos diabéticos e não diabéticos, Foos et al. (1980) demonstraram que o DVP foi significantemente mais comum nos pacientes diabéticos.

Processo hemorrágico e/ou inflamatório vítreo foi encontrado em 2,1\% (dois olhos de um mesmo animal) dos olhos dos cães com catarata e 3,1\% (dois olhos de um mesmo animal) dos olhos dos cães diabéticos. No primeiro caso, após o exame ultra-sonográfico, foi detectada trombocitopenia no paciente, o que contra-indicou, de imediato, a facectomia. No segundo caso, o animal apresentava idade avançada (17 anos), diabetes diagnosticado havia 4 anos, catarata hipermatura e uveíte facogênica nos dois olhos. A cirurgia de catarata também foi contra-indicada nesse paciente. A porcentagem de $2,1 \%$ de hemorragia vítrea no grupo com catarata foi condizente com a literatura revisada, na qual foram encontradas as taxas de $2,5 \%$ e $1,72 \%$ de hemorragia vítrea em pacientes humanos (Lacava \& Centurion 1997, Anteby 1998,).

Hialose asteróide foi observada em $4,6 \%$ no grupo controle, $2,1 \%$ no grupo com catarata e $12,3 \%$ no grupo com diabetes e catarata. Portanto, o grupo de cães diabéticos apresen- 
tou uma porcentagem maior, estatisticamente significante, de hialose asteróide, achados compatíveis com a literatura para pacientes humanos. Rubin (1963) concluiu que os corpos asteróides na hialose asteróide no cão e no homem são quimicamente similares, porém não conseguiu associá-los a doenças sistêmicas. Posteriormente, vários autores correlacionaram a hialose asteróide com o diabetes melito (Wasano 1987, Bergren et al. 1991, Yazar 2001, Akram 2003). Em nosso estudo, houve relação estatisticamente significante entre a presença de hialose asteróide e tempo de evolução do diabetes. Dos animais diabéticos havia mais de dois anos, 38,9\% apresentaram hialose asteróide, enquanto apenas 3,7\% dos animais diabéticos entre 6 meses e dois anos apresentavam tal alteração. Todos os animais com hialose asteróide apresentaram, também, catarata hipermatura. Hialose não foi encontrada em nenhum dos pacientes cujo diabetes foi diagnosticada havia menos de 6 meses. $O$ aparecimento de hialose asteróide está, portanto, evidentemente relacionado com a presença do diabetes melito e com o tempo de evolução da doença.

O diagnóstico de descolamento de retina foi baseado no aparecimento de uma estrutura linear, ecodensa, no segmento posterior, pouco móvel, com mobilidade do tipo "corda de violão", aderida no disco óptico e, na maioria dos casos, na ora serrata. Foi encontrado DR (descolamento de retina) em 4,3\% dos olhos dos cães com catarata (quatro olhos, três com DR parcial e um com DR total) e em 3,1\% dos olhos dos cães diabéticos (dois olhos com DR parcial). Em todos os casos, o DR foi unilateral. Não foi encontrada relação do DR com o tempo de evolução nem com a classificação da catarata em nenhum dos dois grupos, apesar de que, num total de seis olhos com DR, um apresentava catarata imatura, um, catarata matura e quatro, catarata hipermatura. Em todos os casos, a cirurgia foi contra-indicada no olho com DR e foi realizada no olho contralateral. Em estudo realizado com 93 pacientes humanos diabéticos (168 olhos), Jerneld et al. (1980) diagnosticaram, ultra-sonograficamente, DR em $32 \%$ dos olhos. Neste mesmo trabalho, determinou-se que a acurácia do ultra-som no diagnóstico de DR foi de apenas $78 \%$. Uma hipótese referente à baixa freqüiencia de descolamento de retina em cães diabéticos, em relação a pacientes humanos diabéticos, é que o cão desenvolve catarata mais freqüentemente que o ser humano. Essa catarata é intumescente, aumentando o volume do cristalino, o que empurra o vítreo em direção à parede posterior, exercendo pressão sobre a retina e dificultando o seu descolamento.

Em nosso estudo, não observamos evidências de vitreopatia nem de retinopatia diabética, tais como proliferações vítreorretinianas, DRs tracionais ou hemorragias. As alterações de retina em cães diabéticos, em comparação com pacientes humanos, são mais discretas e limitadas à formação de microaneurismas, devido a perda dos pericitos das paredes dos capilares da retina, além de algumas outras alterações morfológicas nas veias retinianas (Basher \& Roberts 1995).

A importância da ultra-sonografia ocular na avaliação préoperatória de pacientes humanos com catarata total foi res- saltada por diversos autores. Lacava \& Centurion (1997) avaliaram os exames de 113 pacientes que foram encaminhados com pré-operatório de facectomia e obtiveram, entre outras alterações, 6,08\% de descolamento de retina e 39,13\% de descolamento de vítreo posterior, citado, pelos autores, como o mais importante evento predisponente ao descolamento de retina. Observou-se, no estudo acima, que cerca de $10 \%$ dos pacientes apresentavam alterações que comprometeriam o resultado funcional pós-cirúrgico, caso a ecografia não fosse realizada no pré-operatório (descolamento de retina, hemorragia vítrea, coloboma, alteração macular e papilar). Um ano mais tarde, outro estudo, com amostragem maior, de 509 pacientes portadores de catarata, foi realizado (Anteby 1998). Encontrou-se alteração de segmento posterior em $19,6 \%$ dos casos, sendo as mais freqüientes: $7,2 \%$ de estafiloma posterior, $4,5 \%$ de descolamento de retina e $2,5 \%$ de hemorragia vítrea.

Corrêa et al. (2002) realizaram estudo semelhante, porém comparando os achados ultra-sonográficos pré-cirúrgicos com o exame pós-cirúrgico dos mesmos pacientes, para avaliar a sensibilidade e a especificidade do ultra-som como método diagnóstico. A concordância entre os achados pré e pós-operatórios foi de 95,4\%. O ultra-som apresentou sensibilidade de $91,3 \%$ e especificidade de $100 \%$, o que confirma a importância desse método diagnóstico na avaliação pré-operatória de pacientes com cataratas densas.

Grottone (2003) conduziu um estudo de repercussão econômica da realização de ultra-sonografia ocular em pré-operatório de pacientes com catarata densa, assistidos pelo Sistema Único de Saúde (SUS). Descolamento de retina foi encontrado em 6,3\%, DVP parcial, em $28,9 \%$, DVP total, em $41,8 \%$ e opacidades vítreas em $34,2 \%$ dos olhos avaliados. Neste estudo, o autor concluiu que os pacientes com catarata densa e menos de 50 anos de idade apresentaram maior freqüiência de descolamento de retina e que a quantidade de recursos financeiros empregados para a realização de ultra-sonografia ocular na amostra estudada foi inferior ao valor estimado para pagamento das cirurgias dos pacientes com descolamento de retina que estavam sem indicação de facectomia, permitindo a otimização do destino da verba pública.

Em cães, poucos estudos foram realizados envolvendo o exame ultra-sonográfico ocular de pacientes com catarata total. Um dos poucos trabalhos em pacientes caninos portadores de catarata foi realizado por Van der Woerdt et al. (1993). Eles avaliaram, retrospectivamente, 147 casos (277 olhos). Desses animais, 23\% apresentavam degeneração vítrea, 9\%, DR e $2 \%$, degeneração vítrea e DR. Os autores encontraram associação significante entre classificação da catarata e DR, porém não houve associação entre classificação da catarata e degeneração vítrea, ou DR e degeneração vítrea.

Em nosso estudo, assim como no estudo supracitado (Van Der Woerdt 1993), não evidenciamos roturas retinianas. É possível que pequenas roturas não sejam detectadas ultrasonograficamente. Além disso, há uma limitação na visibilidade da periferia do segmento posterior devido ao fato de não conseguirmos posicionamento ocular voluntário do cão para os cortes longitudinais. $\mathrm{O}$ transdutor por nós utilizado é 
linear, de 3,5mm, o que também dificulta a visibilidade da periferia ocular.

A ultra-sonografia ocular em cães é um campo muito abrangente, que merece ser amplamente explorado. Estudos adicionais poderão avaliar a sensibilidade e a especificidade do exame em cães, comparando os achados ultra-sonográficos pré-cirúrgicos de catarata com os achados oftalmoscópios póscirúrgicos.

Existe um longo caminho a percorrer em relação à retinopatia diabética em cães. $O$ cão é, certamente, um potencial modelo experimental para o estudo da doença em seres humanos. Porém a ultra-sonografia ocular como exame único pré-cirúrgico não é suficiente para a detecção de todas as alterações retinianas causadas pelo diabetes. Faz-se necessária a utilização de outras ferramentas diagnósticas, como, por exemplo, a eletrorretinografia, que mensura a atividade elétrica da retina, e a angiografia fluoresceínica, que seria capaz de detectar pequenas áreas de hemorragia ou microaneurismas retinianos.

\section{CONCLUSÕES}

Os resultados obtidos na pesquisa, na forma como foi realizada, permitiram concluir que:

- Cães diabéticos têm mais predisposição ao desenvolvimento de hialose asteróide, que ocorre com maior frequiência em animais diagnosticados como diabéticos há mais de 2 anos;

- Não houve diferença na porcentagem de descolamento de retina encontrada em cães diabéticos e não diabéticos;

- A ultra-sonografia ocular é um exame seguro, eficaz e importante na avaliação do pré-operatório de cães portadores de catarata.

Agradecimentos.- Ao Professor Dr. Carlos Eduardo Larsson do Departamento de Clínica Médica da FMVZ, USP, pelo empréstimo do aparelho de ultrasom, permitindo a realização deste trabalho.

\section{REFERÊNCIAS}

Akran A., Niazi M.K., Ishaq M. \& Azad N. 2003. Frequency of diabetics in asteroid hyalosis patients. J. Ayub Med. College, Abbottabad, 15(3):10-11.

Anteby I.I., Blumenthal E.Z., Zamir E. \& Waindin P. 1998. The role of preoperative ultrasonography for patients with dense cataract: a retrospective study of 509 cases. Ophthalmic Surg. Lasers 29(2):114-118.

Banett K.C. 1981. Diabetic retinopathy in the dog. Brit. J. Ophthalmol. 65(5):312-314

Basher A.W.P. \& Roberts S.M. 1995. Ocular manifestations of diabetes mellitus: diabetic cataract in dogs. Vet. Clin. North Am. Small Anim. Pract. 25(3):661676 .

Bergren R.L., Brown G.C. \& Duker J.S. 1991. Prevalence and association of Asteriod Hyalosis with systemic diseases. Am. J. Ophthalmol. 111(3):289293.
Correa Z.M.S., Goldhadt R., Marcon A.S. \& Marcon I.M. 2002. Achados ecográficos em pacientes com catarata total. Arqs Bras. Oftalmol. 65:609613.

Feldman E.C. \& Nelson R.W. 1987. Diabetes mellitus, p.261-294. In: Idem (ed.), Canine and Feline Endocrinology and Reproduction. W.B. Saunders, Philadelphia,

Foos R.Y., Kreiger A.E., Forsythe A.B. \& Zakka K.A. 1980. Posterior vitreous detachment in diabetic subjects. Ophthalmol. 87(2):122-128.

Grottone G.T. 2003. Repercussão econômica da realização de ultra-sonografia ocular em pré-operatório de pacientes com catarata densa, assistidos pelo Sistema Único de Saúde - SUS 2003. Tese de Mestrado Profissionalizante em Administração da Prática Oftalmológica, apresentada à Escola Paulista de Medicina, Universidade Federal de São Paulo, São Paulo. 51p.

Jerneld B., Alguere P. \& Singh G. 1980. Ultrasonography of diabetic vitreous disease with low visual acuity. Acta Ophthalmol. 58(2):193-201.

Lacava A.C. \& Centurion V. 1997. Impotância da ultra-sonografia ocular na avaliação pré-operatória da facectomia. Revta Bras. Oftalmol. 56(8):603606.

Landrey M.P., Herring I.P. \& Panciera D.L. 2003. Diabetic retinopathy in dogs following lensectomy by phacoemulsification: 52 cases (1993-2003). 34th Annu. Meet. Am. Coll.Vet. Ophthalmol., Coeur D'Alene, ID, USA, p.16. (Abstracts)

Monti F., Bellan B., Berardi S. \& Peruccio C. 1976. The clinical picture of diabetic retinopathy in the dog. Folia Vet. Latina 6(3):249-274.

Oksala A. 1975. Ultrasonic findings in the vitreous body at different ages and patients with detachment of the retina. Albrecht von Graefes Arch. f. Klin. Exp. Ophthalmol. 197(1):83-87.

Oksala A. 1978. Ultrasonic findings in the vitreous body at various ages. Albrecht von Graefes Arch. f. Klin.Exp. Ophthalmol. 207:275-280.

Ono K., Yasuda K., Iwata H., Nakayama H., Hasegawa A. \& Tomoda I. 1986. Fluorescein angiogram in diabetic dogs. Jap. J. Vet. Sci. 48(6):1257-1261.

Ossoinig K.C. 1979. Standardized echography: basic principles, clinical applications, and results. Int. Ophthalmol. Clinics 19:127-210.

Patz A., Berkow J.W. \& Maumenee A.E. 1965. Studies on diabetic retinopathy. II. Retinopathy and nephropathy in spontaneous canine diabetes. Diabetes 14:700-708.

Patz A. \& Maumenne A.E. 1962. Studies on diabetic retinopathy. I. Retinopathy in a dog with spontaneous diabetes mellitus. Am. J. Ophthalmol. 54:532541.

Rossi A. \& Gallenga P.E. 1971. Ultrasonographic features of the senile vitreous body, p.247-253. In: Böck J. \& Oissonig K. (ed.), Ultrasonographia Medica. Vol.2. Wiener Medizinische Akademie, Wien.

Rubin L.F. 1963. Asteroid hyalosis in the dog. Am. J. Vet. Res. 24(103):12561262.

Sibay T.M. \& Hausler H.R. 1967. Eye findings in two spontaneously diabetic related dogs. Am. J. Ophthalmol. 63:289-294.

Van der Woerdt A., Wilkie D.A. \& Myer C.W. 1993. Ultrasonigraphic abnormalities in the eyes of dogs with cataracts: 147 cases (1986-1992). J. Am. Anim. Hosp. Assoc. 203(6):838-841.

Wasano T., Hirokawa H., Tagawa H., Trempe C.L. \& Buzney S.M. 1987. Asteriod hyalosis: posterior vitreous detachment and diabetic retinopathy. Ann. Ophthalmol. 19(7):255-258.

Wilkinson J.S. 1960. Spontaneous diabetes mellitus. Vet. Rec. 72:548-555.

Yazar Z., Hanioglu S., Karakoc G. \& Gursel E. 2001. Asteriod hyalosis. Eur. J. Ophthalmol. 11(1):57-61. 